\title{
Increase of Safety Use Robots in Industry 4.0 by Developing Sensitivity and Professional Behavioral Skills
}

\author{
Evgeniy Bryndin \\ Scientific Department, Research Center "Natural Informatic", Novosibirsk, Russia
}

Email address:

bryndin15@yandex.ru

To cite this article:

Evgeniy Bryndin. Increase of Safety Use Robots in Industry 4.0 by Developing Sensitivity and Professional Behavioral Skills. American Journal of Mechanical and Industrial Engineering. Vol. 5, No. 1, 2020, pp. 6-14. doi: 10.11648/j.ajmie.20200501.12

Received: May 26, 2020; Accepted: June 5, 2020; Published: June 23, 2020

\begin{abstract}
The paper highlights general approach to creating cognitive adaptive robots with imitative thinking and adaptive behavior as an assistant to humans in various fields of activity. Communicative-associative symbolic-language thinking logic allows the creation of cognitive adaptive robots for different spheres of life. Specialization of cognitive adaptive robots is carried out on the basis of knowledge bases, bases of abilities and implementers of behavior. Adaptive behavior is guided by imitative thinking on a human task and is carried out by models of environment and behavior. Specialization of the robot is set by the subject knowledge base and base of abilities, and equipment (sensors, sensors, manipulators, motor systems and other devices). For collaborative robotics, you need a soft, sensitive, and naturally highly intelligent solution. The focus here is on pneumatic light robots, which are inherently designed to work together and can present a cost-effective alternative to humans in the future. The cognitive robot, by increasing visual, sound, substantive, spatial and temporal sensitivity, is able to acquire new skills and accumulate experience of behavior with the necessary technical means. New skills are taught by a retraining system in virtual space, and then the robot develops them in a specific environment. Thus, the professional experience of the cognitive robot in various specific environments is gradually accumulated and its sensitivity is increased. Cognitive adaptive retractable robots with imitative thinking, adaptive behavior, and increased sensitivity have the prospect of widespread practical application.
\end{abstract}

Keywords: Sensitivity, Professional Retraining, Adaptive Behavior, Programming of Basic Functions, Safety

\section{Introduction}

Robots can solve many different practical problems. Medicine, banking, industry, education, hotel business and even entertainment are the main applications of robots. Health care is one of the most progressive areas in which robot labour is used. Robotic surgery is currently actively developing. In medicine, a great breakthrough has been achieved since bionic prostheses, which a person can manage with his own nervous system, began to be used. Moreover, with the help of a prosthesis, a person can feel touch, heat and pressure.

Robotic systems are used in the field of security: devices with special sensors quickly detect fire-hazardous situations and successfully prevent them. Modern factories and enterprises have advanced far at the expense of modern technologies. Automated industrial robots are used for welding, laying, painting and other operations requiring repeated and high accuracy. Robots for high-tech work are emerging, which is on the teeth of artificial intelligence.

Cosmonautics robots are actively used by man in mastering the spaces of the universe. They collect soil samples and explore new spaces under conditions of increased radiation and extreme temperatures. Russian Robot FEDOR (Final Experimental Demonstration Object Research) - an anthropomorphic robot is a Skybot F-850 astronaut.

In Japan, Russia, Taiwan, China and other developed countries, androids have been created that can maintain conversation and even joke. With the advent of artificial neural networks in the modern world, robots have learned to create. In modern life, man already uses robots in all spheres of his activity. In most cases, robots are not replacement assistants. Man is trying to create, for his robots, artificial intelligence. With artificial intelligence, robots will be able to independently assess what is happening around them and 
make decisions on the actions they need to take.

The development of artificial intelligence and machine learning technologies and their application in robotics is a prerequisite for the creation of really useful and smart robots. Statistical methods and machine learning, including artificial neural networks of deep learning, have had a huge impact on modern robotics. The architecture of networks is becoming more complex and capacity is increased while maintaining an acceptable learning speed, as well as the development of systems that will allow neural networks to operate with minimal energy consumption. An important task in improving the efficiency of machine learning is to reduce the learning sample while maintaining the speed and quality of learning. Training in action algorithms begins. A neural network trained on the example of a single labeled dataset can self-train and draw conclusions on unmarked datasets. As a result, learning becomes faster, large amounts of data are processed, and the quality of results is improved.

Current robotics practice shows that the best results in increasing productivity can be achieved from the maximum efficiency of the bundle of robot teams and people working together to achieve a common goal. Increase social interaction between humans and robots in everyday and working life is the subject of numerous studies, some of which have become the basis of as many billions of industries. An example of successful implementation of social interaction technologies is voice assistants and chat bots.

Robots can already both record human movement skills and copy them. Machine learning improves drive efficiency and mobility. As a result, more complex movements will be achieved by simpler means. Now the developments in this direction are carried out by Boston Dynamics and MITs with the robot Atlas. Researchers hope that if successful, the application of neural networks will find new variants of movements that will be more effective. In the coming years, the quality of training will improve and the degree of autonomy of robots will increase

The paper briefly discusses the approach to creating retractable cognitive sensitive robots capable of working in the high-tech labor market.

\section{Sensitive Aspects of Cognitive Robot}

Cognitive robot with visual, sound, subject, spatial and temporal sensitivity solves many professional problems [1].

The subject sensitivity of a robot is the ability to determine the properties, characteristics, and qualities of objects using tactile and kinesthetic sensor sensors designed to measure the characteristics of the external environment. The solution of many problems related to the search for objects, their capture and manipulation was made possible only with the development of sensors with tactile and kinesthetic sensitivity. The simplest type it are contact sensors. They are micro switches that record contact with the object. Tactile sensors allow you to respond to touch and measure the pressure at the points where the sensor contacts the object.
They are usually placed on the bumpers of transport robots or on the grip of manipulation robots. These sensors serve to detect individual objects, prevent damage to these objects and the robot itself, and to recognize the external environment by contacting and sensing. Kinesthetic sensors detect position, movement of actuators (for example, fingers of manipulator grip) and forces arising in them. An important feature of tactile and kinesthetic sensors is their ability to operate in almost any environment. In particular, these sensors are indispensable for underwater robots, as when water is clouded, the television or optical feedback channel stops working.

The robot can maintain vital human heat and cold parameters within acceptable limits. Pressure is a function that a professional robot needs. This function can be performed by the robot by various methods and devices depending on its purpose. This is especially important when the robot's fingers must capture a variety of, sometimes fragile, objects. Touch sensors in the robot must be sensitive and numerous, allowing them to be used to distinguish shape.

The robot reproduces the smell by technical means. When the combustion mixture of gas and air comes into contact with some catalysts, such as platinum or palladium, heat is generated and the electrical resistance of the catalyst is changed, which can be detected by direct measurement. Such detectors are very important for preventing fire and explosion. Oxygen can be detected using its paramagnetic properties, and some gases can be detected using its thermal conductivity, in a device called a thermo computer metric detector. Infrared absorption can also be used to detect various gases. Water vapor is detected by hygrometers of various types. Gases have the ability to change the color of various chemicals, which is often used in gas analysis. A similar method can be applied to a robot. In Japan, a gas trap device "Taguchi gas sensor" has been developed. Solid state technology has made it possible to manufacture an oxygen analyzer that can be used to determine the concentration of oxygen in flue gases. Future research will lead to better odour detectors.

Various elements are used to sense humidity, including lithium chloride (Dunmore sensor), carbon, polyelectrolytebased elements, ceramic elements, capacitive devices and alumina-based elements. These developments can be used in robotics.

The robot is capable of measuring the temperature level either within itself or in the environment. For this purpose, any of the well-known methods of electrical temperature determination are used.

The method of accurate indication of robot position is implemented by navigation technique based on comparison of time positions of pulses with time positions of reference pulses. Indicating the position of a robot is important for orienting it in space. Object image mapping and localization is carried out using scanning laser range finders. Ultrasonic sonars, infrared sensors, laser sensors are used to measure distance. The object is reached by the obtained coordinates. The behavior implementation procedures perform the 
movements of the parts of the robot motor mechanisms in accordance with the movement route and the sequence of motor acts of the parts of the robot motor mechanisms. The robot movement route is built in classes of piece-polynomial functions.

The implementation of visual sensitivity relies on obtaining information about different parts of the object independently of each other. Scanning with two brightcentered lasers, you go through the entire sample and see a very thin structure with resolution in nanometers. The detail of the information received is increasing. At present, overresolution optical nanoscopy can be used to implement frequency spectral vision of robots with expansion of their spectral range, spectral and spatial resolution. This requires the integrated use of several instruments operating in different spectral bands. Use the possibilities of combined application of monophotonic UV-C sensor and hyperspectration modules of visible near infrared range. Sensor UV-C is able to quickly detect the object of interest and transmit its coordinates to the control unit to guide the hyperspectometer to the target and its detailed shooting with high spectral and spatial resolution. In multi-spectrum enhanced vision systems, choosing a strategy for combining information from multiple video channels plays an important role. The robot's spectroscopic vision helps it perceive the frequency spectra of objects and objects of the environment. Practical application of hyperspectration sensors of visible and near infrared range covers a wide range of tasks of science and national economy as: geology, agriculture, forestry and water economy, ecology, urban infrastructure and many others. CNN's twist neural networks are an effective tool for detecting and classifying objects. An artificial trained neural network recognizes their spectrum, associatively compares with accumulated spectra of objects and objects in its frequency base. By associative comparison, determines the object or object [2].

The sound sensitivity of the robot is realized by sound sensors. Sound sensors include a variety of microphones and ultrasonic sensors. The microphones serve to sense sound commands during voice control of the robot. Ultrasonic sensors consist of a transmitter and a signal receiver. With the ultrasonic signal reflected from the objects, they can detect them and determine the distance to them. Ultrasonic sensors have advantages over optical sensors. They can detect transparent objects; their readings are independent of lighting conditions and are not sensitive to changes in the physical properties of the environment; the service life of vibration generators is almost unlimited, etc. However, due to the fuzzy direction of the ultrasonic vibrations, the accuracy of determining the distances to the objects of such sensors is low. In addition, they are unable to detect objects with very small dimensions, which is due to the relatively long length of ultrasonic waves.

The motor activity of a cognitive robot is related to the performance of a variety of speed and force actions. The ability to correctly distribute efforts in time and space is one of the main conditions for the manifestation of professional technical skill.

In many cases, the differentiation of forces by magnitude, place and time of their manifestation largely determines the class of the robot, which manifests itself in the qualitative performance of the movement, most determined by the level of force distinction, accuracy of actions, fine differentiation of the forces of the manipulator brush.

The sensitivity of robots of different specializations is determined by the following general patterns:

1. quality and accuracy of effort differentiation are improved in the process of targeted training with increase of accuracy of time and space perception;

2. development of distinctive sensitivity of efforts in a specific way in different types of activities;

3. level of power sensitivity development depends on selfcontrol.

Training of distinctive sensitivity of force is performed by method of minimum force increment. The number of force stages characterizes the sensitivity of force training.

Modern devices, cybernetic techniques, and artificial intelligence enable the creation of cognitive robots with adaptive behavior based on imitative thinking with professional diversification and mobility.

\section{Management of Behavior of the Robot}

Robot behavior is controlled by a polyfunctional hierarchical system of controllers similar to the system of controlling human body behavior. Behavior occurs under the influence of an information requirement (task), which causes orientation in the situation by models of environment and behavior. The orientation is completed by selecting the appropriate hierarchical controller system program, the execution of which results in the implementation of the behavior. The controller is the computer on the chip. It is designed to control various electronic devices. The robot controller is the smallest computer. It contains a processor and peripheral devices: FLASH memory, timers, interfaces for communication with external devices and many other useful circuits. The controller operates according to a given program, which is loaded into it from a powerful computer. It is loaded by a programmer into the microcontroller. The microcontroller can process the information from the inputs and generate electrical signals at the outputs according to how we program the behavior of the robot [3].

The robot has sensors to interact with the environment that get information from the environment. For example, phototransistor, microphone, contact sensor.

A hierarchical approach to implementing the behavior actions of a cognitive mobile robot allows it to perform useful work and ensure its movement. Hierarchical algorithms of behavior actions are divided into agglomerative and divisional ones. Agglomerative algorithms begin their execution by putting each action into a corresponding cluster and, as executed, combining the clusters, until one cluster including all behavior actions is finally received. Division algorithms, by contrast, first assign all actions to a single 
cluster and then divide that cluster until each action is in the corresponding cluster. The representation of the result of the hierarchical algorithm is a dendrogram - a diagram showing in what sequence the actions were merged into a cluster or the actions were divided into clusters.

This approach makes it possible to formalize the mobility requirements of the robot behavior and to develop all possible algorithms for responding to changes in the state of the environment. For example, when moving on the street, using satellite navigation technology, and surrounding objects, detecting with cameras or range meters. That is, the approach allows autonomous robotics systems to be designed for the implementation of many industrial and social spheres of life.

\section{Programming of Behavior of the Robot}

The behavior of the robot is set in programming languages. Many programs for the generalizing architecture were realized in behavior language which was defined by Brooks. This language represents language of management in real time on the basis of rules which result of compilation are AFSM controllers. The separate rules of this language set by means of syntax, similar to Lisp are compiled in the AFSM submachine guns, and groups of the AFSM submachine guns unite with the help of set of mechanisms of transfer of local and global messages.

As well as the generalizing architecture, language of behavior is restricted as it is aimed at creation of the simple AFSM submachine guns with rather narrow definition of a stream of communication between modules. But recently on the basis of this idea new researches which led to creation of a number of the programming languages similar on the spirit to behavior language, but more potent and providing faster realization are conducted.

One of such languages is the universal robotic language, or in abbreviated form GRL (Generic Robot Language). GRL is a functional programming language for creation of larger modular control systems. As well as in behavior language, in GRL as the main design units finite-state automations are used. But as control over these automatic machines the GRL language offers much wider list of designs for definition of a communication stream and synchronization of restrictions between various modules, than behavior language. Programs in the GRL language are compiled in efficient programs in such languages of teams as Page.

One more important programming language (and the related architecture) for the parallel robotic software is the system of scheduling of jet actions, or in abbreviated form RAPS (Reactive Action Plan System). The RAPS system allows programmers to set the purposes, plans, the bound to these purposes (or partially to define policy) and also to set conditions under which these plans most probably will be implemented successfully.

Extremely important the fact that also the tools allowing to cope with inevitable refusals which arise in actual robotic systems are provided in the RAPS system. The programmer can set procedures of detection of refusals of various types and provide the procedure of elimination of an exclusive situation for each type of refusal. In three-level architecture the RAPS system is often used at the executive level that allows cope with the unexpected situations which are not demanding rescheduling successfully.

There are also several other languages which provide use in robots of means of formation of reasonings and tutorials. For example, Golog represents the programming language allowing provide perfect interaction of means of algorithmic problem solving (scheduling) and the means of jet management set immediately by means of the specification.

Programs in the Golog language are formulated in terms of situational calculation taking into account a padding possibility of application of operators of nondeterministic actions. Except the specification of a time schedule control with opportunities of nondeterministic actions, the programmer has to provide also the complete model of the robot and its environment.

As soon as the time schedule control reaches a point of the nondeterministic choice, the scheduler (the given in a form of the program of the theorem proving) for definition of what to do next is called. Thus, the programmer can partially define the given controllers and rely on use of the firmware schedulers for acceptance of the final choice of the management plan.

The main attractive feature of the Golog language is the perfect integration of means of jet management and algorithmic management provided in it. In spite of the fact that when using the Golog language it is necessary to keep rigorous requirements (the complete observability, discrete states, the complete model), by means of this language highlevel controlling means for a number of the mobile robots intended for application in rooms were created.

Language "JSk CES (reduction from $\mathrm{C}++$ for embedded systems $-\mathrm{C}++$ for the firmware systems) is a language expansion $\mathrm{C}++$ in which probability tools and tutorials unite. Probability distributions are among types of data of CES that allows the programmer to carry out calculations with use of acritical information, without spending those efforts which are usually bound to realization of probability methods.

Even more important the fact that the CES language provides setting up the robotic software by means of training at the basis of examples, in many respects similar to what is carried out in tutoring algorithms. The CES language allows programmers to leave in the intervals code which are filled with the training functions; usually such intervals are differentiable parametrical representations, such as neuronic networks. Further at separate grade levels for which the teacher has to set the required output behavior there is the inductive tutoring by means of these functions. Practice showed that the CES language can successfully be applied in problem areas, the characteristic of partially observed and continuous environment.

The ALisp language represents expansion of the Lisp 
language. The ALisp language allows programmers to set the nondeterministic points of the choice similar to choice points in the Golog language. But in the ALisp language not the program of the theorem proving, but means of definition of the exact action by means of the inductive tutoring in which tutoring with a reinforcement is used is applied to a decision making. Therefore the ALisp language can be considered as a convenient way of introduction of knowledge of problem area in the procedure of tutoring with reinforcement, especially knowledge of hierarchical structure of "procedures" of desirable behavior. Still the ALisp language was applied to problem solving of robotics only in imitating researches. It can be finished for programming of robots with imitative thinking and adaptive behavior, capable to tutoring as a result of interaction with the environment.

Sensitivity, combined with sufficiently advanced and diverse artificial intelligence control software, allows cognitive robots to work with undirected objects of arbitrary shape, to assemble and assemble structures according to the drawing, to interact with the external environment, to perform the required sequence of operations in a changing environment. The sensitivity of cognitive robots with artificial intelligence is a necessary prerequisite for enhancing their functional and motor professional capabilities.

Cognitive robots with imitative thinking and adaptive behavior have the prospect of widespread practical application as digital smart robot lecturers and consultants in the educational activities of digital universities to teach students based on online courses. Cognitive robots with imitative thinking and software interface can be used by managers and programmed to control robotic smart factories [4].

\section{Structural Aspects of the Retractable Cognitive Robot}

The cognitive robot has the system of recognition of objects, the system of speech input of information requirements, the system of realization of information requirements (the system of imitation of imitative thinking), the neural network system of synthesis of the speech in the text of realization of information requirement.

The information demand realization system comprises a knowledge acquisition system, a symbolic-language communication system, a training system, a knowledge base, a skills base, a neural network reading system, a printing system and a graphic display system. The training system comprises automatic translation subsystems. The object recognition system is a neural network system.

The robot is introduced to humans through a neural network recognition system. If a person is unknown to a robot, the recipe system remembers his speech dictionary and face. If it is known to the robot, the system will configure the voice input system of the information demand to the voice dictionary of the friend. After that, information contact between the robot and the person begins. The voice input system converts the voice information requirement into text in a functional natural language. A robot based on symbolic language communicative logic with associative and communicative symbolic language elements of knowledge models imitative thinking [5].

The cognitive architecture of the robot develops functional activity (Figure 1).

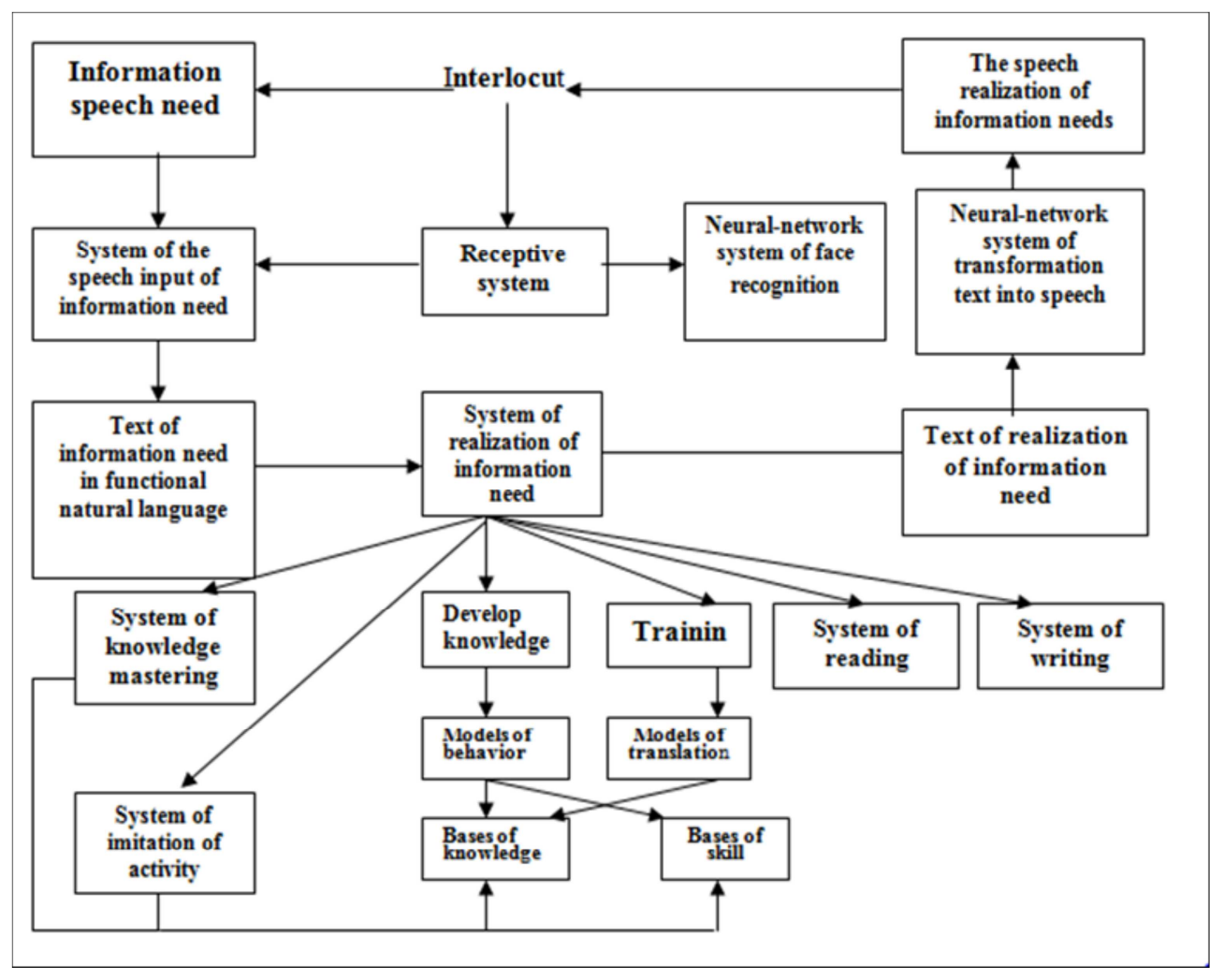

Figure 1. The Architecture of the Retrained Cognitive Robot. 
The robot's smart cognitive architecture determines step by step how best to achieve set goals and realize preferences through utility function, actions based on qualitative selection. Professional self-improvement is carried out by machine re-training according to the criterion of preferences on the basis of extensive statistics of qualitative selection of accumulated innovative skills and competences in the sixth technological order of the industry 4.0 [6-7]. The robot's intelligent cognitive architecture develops its artificial intelligence through machine retraining, based on extensive statistics of innovative competences accumulated in the knowledge base and in the skills base of relevant professional and behavioral skills.

The information unit of communication between person and robot is the information need. The contact uses the information requirements contained in the robot knowledge base, as well as combinations of information requirements. The robot receives new information needs and their implementation during its training. Multi valued words of information need have meaningful markings. Cognitive navigation of the robot for its implementation is carried out through entity dictionary. For example, the sense markings of the word "fold" indicate or lexical value, or arithmetic action, or behavioral action.

\section{Information Demand Realization System}

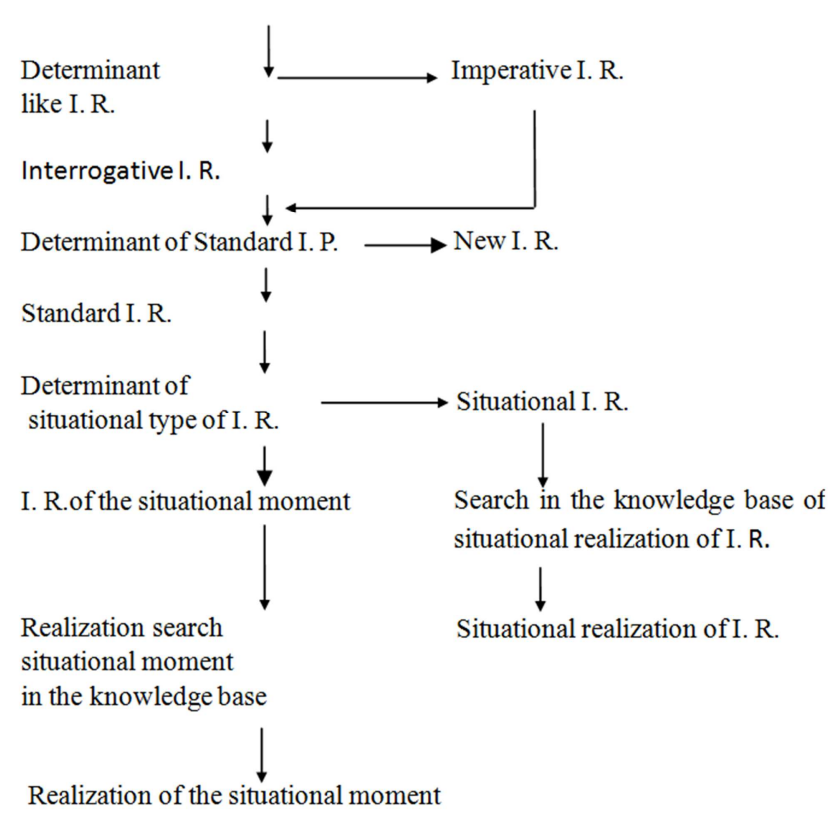

Figure 2. Situation Information Needs Implementation Diagram.

The information requirement (job) is composed of knowledge elements. Information needs are stimulating and questioning. An incentive information requirement consists of an information action and an information condition. If the information requirement is a question, it clearly indicates the question phrase defining the direction of implementation.
The implementation system includes a knowledge base and a skills base. The realization of the information requirement is taken either from the knowledge base, or is developed by the standard procedure of the implementation of the knowledge base according to the current information requirement, or a network of element-by-element implementation according to the combined information requirement is formed. Schemes for the realization of information need are derived from a study of educational practice in the formation of imitative thinking. The diagrams are shown in Figures 1-2.

Once implemented, the new information need is embedded in the knowledge base and associated with the knowledge element that is its implementation. Variation of such information needs is carried out in variable part, are realized according to common pattern for them by standard procedures.

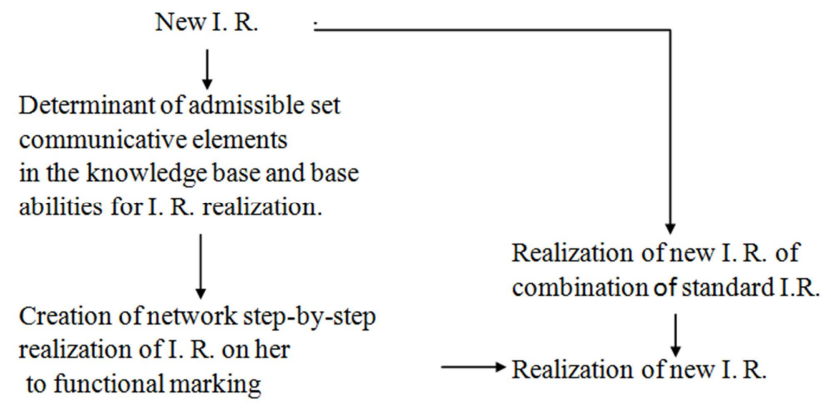

Figure 3. New Information Requirement Implementation Diagram.

The implementations of the combined information needs are generated by investing, varying and combining the implementations of the information needs that are in the knowledge base of the system. New information needs are built by either a sequential merger from realizations of existing needs, an investment in one another, or a merger and an investment at the same time.

\section{Specialization of Cognitive Adaptive Sensitive Robots}

Specialization of cognitive adaptive robots is carried out on the basis of knowledge bases, bases of abilities and implementers of behavior. Adaptive behavior is guided by imitative thinking on a human task and is carried out by models of environment and behavior. Cognitive behavior management is supported by telecommunications management. The adaptive behavior of robots is programmed through a software interface.

A robot component implementing adaptive behavior in an external environment includes a sensory, control, executive motor system and a diagnostic system.

The sensor system is designed to sense and transform information on the state of the external environment. It includes television and optical laser devices, ultrasonic range finders, tactile and contact sensors, position sensors, neural network devices for recognizing images of external environment, etc. Robotic perception is a process in which 
robots display the results of sensory measurements on internal structures of medium representation.

In the process of retraining a new professional activity, the cognitive robot accumulates a basic set of task implementations in the knowledge base [7]. And also accumulates programs of professional action functions in the skills base. By means of more perfect sensors, sensors, controllers and other devices the cognitive robot expands behavioural skills. Based on a basic set of job implementations, the cognitive robot can implement combined jobs. See industrial robotic complexes of different countries in figures 4-8.

Based on smart big data accumulated in the course of professional activity, can implement new tasks (figures 9-10). To do this, the 4.0 industry attracts digital process twins using a huge array of manufacturing process data. By digital twin is meant a set of digital technologies that use approaches of statistical analysis, machine learning, chemistry, physics, control theory, reliability theory, mass service theory, numerical modeling, optimization, simulation modeling. Machine learning technologies are also involved in digital twins. They are essentially self-learning systems that use information from a range of sources, including data from sensors monitoring various performance indicators of a physical object, information from expert experts and other similar machines or machine parks, and larger systems of which the observed physical object may be a part.

The accumulation of professional and behavioral skills by a cognitive robot increases its visual, substantive, spatial, temporal, and occupational sensitivity.

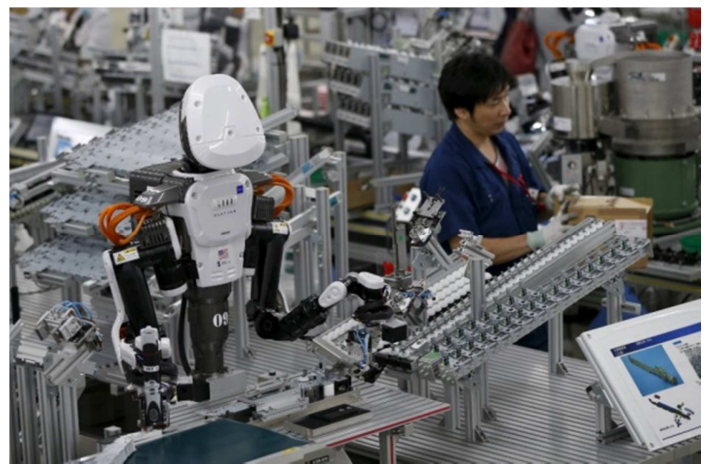

Figure 4. Japanese Industrial Robotic Enterprise.

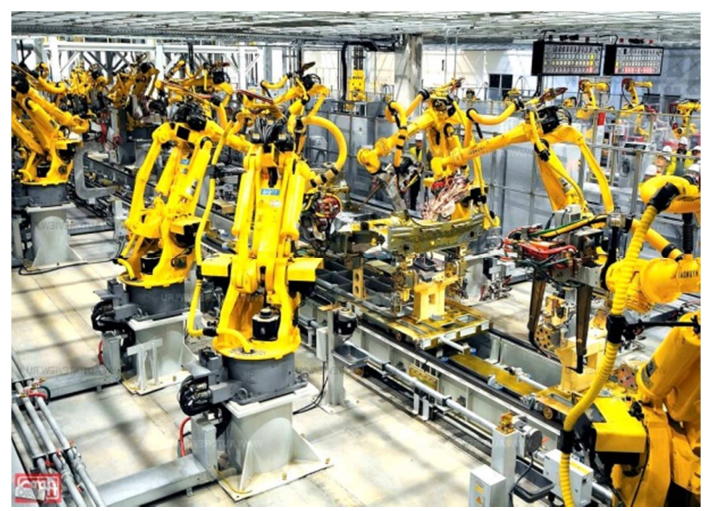

Figure 5. Chinese Industrial Robotic Enterprise.

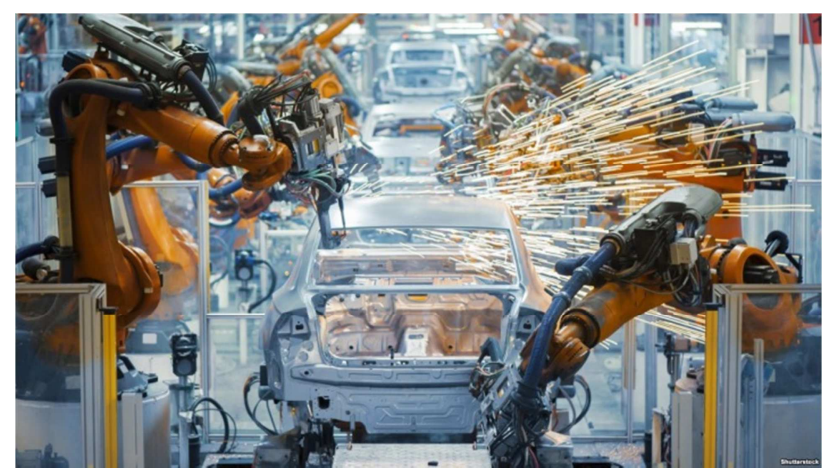

Figure 6. Korean Robotic Automotive Industry.

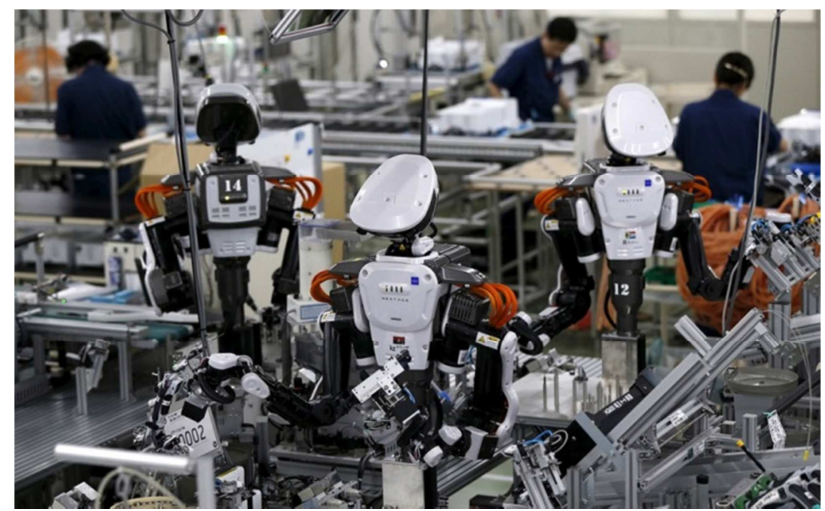

Figure 7. American Industrial Robotic Enterprise.

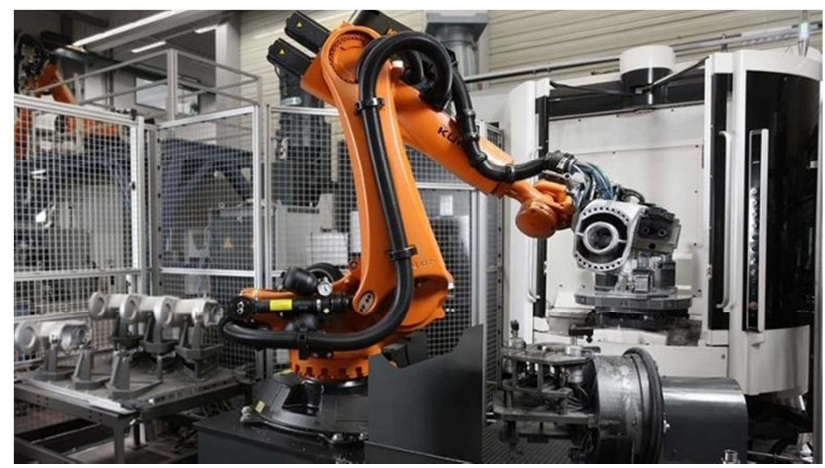

Figure 8. Russian Robotic Metal Processing Enterprise.

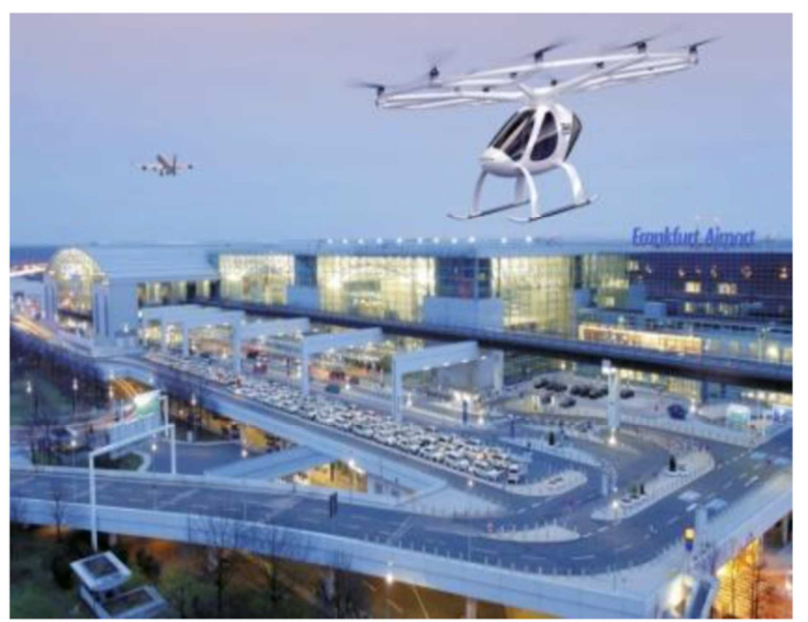

Figure 9. German aerial unmanned taxi. 


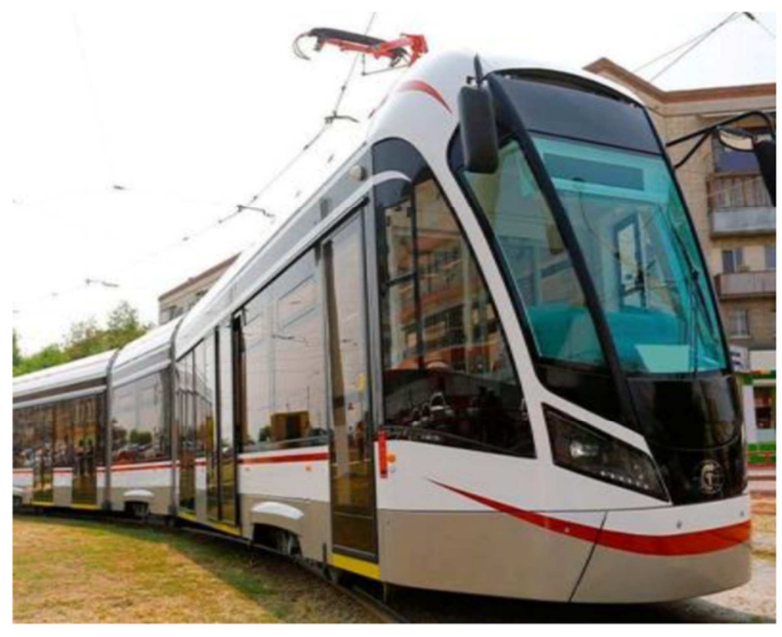

Figure 10. A Russian tram without a driver.

\section{Approach to the Safe use of Intelligent Robots}

Modern artificial intelligence techniques (neural networks, machine learning) and science cannot explain how a trained system makes a decision. Because of this, the price of error in the fields: medicine, defense, judicial practice, etc. Artificial intelligence specialists adjust artificial neural networks with the help of coefficients during their training with framed data to obtain the necessary result. In order to safely use an artificial neural network, it is necessary to determine the range of change of attributes of input data.

In order to apply safely everywhere, it is necessary to introduce standardization of the use of intelligent robots. Briefly we will consider approach to standardization on use of intelligent robots in ISO/IEC 22989 - Information Technology - Artificial Intelligence - Artificial Intelligence Concepts and Terminology.

It is necessary to add the term and definition of intelligent robot to the standard: intelligent robot is complex of compatible intelligent agents interacting through an intelligent interface, implementing technological process, social services, multidisciplinary interdisciplinary research, or production cycle

The following types of classification should be added to the section relating the types of classification to the stages of the life cycle of the system with artificial intelligence

a) diversification,

b) mobility.

The terms and definitions of diversification and mobility should be added to the standard:

diversification - expansion of artificial intelligence functions and mastering of a new type of functionality in order to increase efficiency, quality and functional diversity of the intellectual system;

mobility is the ability of an intelligent robot to rapidly functional retraining and develop its intelligence.

The definitions of diversification and mobility are consistent with the definitions of the standard:

a) life cycle: Development of a system, product, service, project or other human-created entity from design to write-off;

b) life cycle model: The structural basis of life cycle processes and actions, which also serves as a common reference for linking and understanding.

Images and numbers are taken as simulation data. The environment is perceived through images and scenes. Scenes consist of a number of images. Scenes are static (paintings) and dynamic. Dynamic scenes are characterized by patterns of behavior of objects and objects. Patterns are either described by formulas or represented by a graph (numerically). Safety of behavior depends on spatial, temporal, substantive, visual and audible sensitivity. Behavior security is provided within image similarity boundaries in a safe environment. Simulation shows that the development of behavioural skills (mobility) and professional skills (diversification) increases sensitivity of environmental perception, reduces risks, increases safety.

It is necessary to adjust the definition of artificial intelligence of the standard: artificial intelligence is the ability of a system to acquire, process, apply, and diversify knowledge based on prior experience with specific problems related to data attribute processing and intelligent system mobility

Attributes of data. Objects, objects, materials, things, processes, phenomena and other aspects of the physical world have different properties and characteristics. Properties are represented by qualitative attributes. Characteristics are represented by meaningful attributes. A qualitative attribute can be visual or audible. A meaningful attribute can be represented by a number, a language sense value, a visual or audible way, a mathematical or behavioral action, or an algorithm. Meaningful qualitative attributes are big intelligent data of artificial intelligence [8-16].

Big Smart Data (Big SD) is collection of qualitative and quantitative attributes related in time, space, and subject area.

Smart data attributes (numbers and images) form a world view. Big SD attributes of the areas of economics, industrial industries, technology, and professions help build and train artificial neural multilayer artificial intelligence networks to manage, make decisions, and make recommendations to professionals and executives. Applied research modeling helps accumulate Big SD scientific attributes in real time and simultaneously use them to deeply train multi-layer artificial neural control networks to simulate applied research, make decisions, and make recommendations to researchers. By modeling, the optimal (equilibrium safe) state of artificial neural networks of intelligent agents and the limits of attribute values relative to this state are determined. Numeric limits are defined for numeric attribute values. For visual and sound images, the limits of similarity to the optimal image are revealed.

The development and use of equilibrium safety systems can be applied as criterion of companies developing 
intelligent robots.

\section{Conclusion}

The international scientific engineering community is gradually moving towards the technical implementation of a cognitive professional sensitive safe robot with qualification retraining. In the future, in the labor market, cognitive sensitive robots with retraining will perform hightech professional work in various areas of industry and social services. Man will engage in scientific research and innovation, and teach cognitive robots with artificial intelligence to increase the intellectual to recursive selfimprovement to the technological singularity of the artificial mind. Self-improving cognitive robots and artificial neural networks that process numerical data, as well as visual and sound images, will be able to search for patterns in large amounts of data, recognize objects and people, read texts, make decisions, control movements and others. A cognitive sensitive robot with an artificial mind will be able to train, speculate, apply new knowledge and experience of behavior, and respond wisely and safely to the external environment. An artificial mind with technological singularity will be a good aid to humanity in the safe mastering of life spaces.

\section{References}

[1] Evgeniy Bryndin. Development of sensitivity and active behavior of cognitive robot by means artificial intelligence. International Journal of Robotics Research and Development. VOL - 10, ISSUE - 1; 2020. Pages: 1-11.

[2] Evgeniy Bryndin. Robots with Artificial Intelligence and Spectroscopic Sight in Hi-Tech Labor Market. International Journal of Systems Science and Applied Mathematic, V. 4, № 3, 2019. Pages: 31-37.

[3] Evgeniy Bryndin. Mainstreaming technological development of industrial production based on artificial intelligence. COJ Technical \& Scientific Research, 2 (3). 2019. Pages: 1-5.

[4] Evgeniy Bryndin. Program Hierarchical Realization of Adaptation Behavior of the Cognitive Mobile Robot with Imitative Thinking. International Journal of Engineering Management. Volume 1, Issue 4. 2017, pp. 74-79

[5] Evgeniy Bryndin. Cognitive Robots with Imitative Thinking for Digital Libraries, Banks, Universities and Smart Factories. International Journal of Management and Fuzzy Systems. V. 3, N. 5, 2017, pp 57-66.
[6] Evgeniy Bryndin. Communicative Associative Logic of Cognitive Professional Robot with Imitative Thinking. Journal Engineering Mathematics, Volume 2, Issue 2. 2018. Pages: 7985.

[7] Evgeniy Bryndin. Cognitive professional robots with retraining skills. Materials of the III International Conference "Cognitive Robotics. TSU publishing House. 2018. Pages: 3-6.

[8] Evgeniy Bryndin. System retraining to professional competences of cognitive robots on basis of communicative associative logic of technological thinking. International Robotics Automation Journal. 2019; 5 (3): 112-119

[9] Evgeniy Bryndin. Collaboration of Intelligent Interoperable Agents via Smart Interface. International Journal on Data Science and Technology, Vol. 5, Issue 2. 2019

[10] Evgeniy Bryndin. Digital technologies of the industry 4.0. / Chepter 10, C. 201-222, Book: Computer Science Advances: Research and Applications. USA: Nova Science Publisher. 2019. 252 pages.

[11] Evgeniy Bryndin. Supercomputer BEG with Artificial Intelligence of Optimal Resource Use and Management by Continuous Processing of Large Programs. International Journal of Research in Engineering, Vol. 1, Issue 2, 2019. Pages: 9-14.

[12] Evgeniy Bryndin. Development of Artificial Intelligence by Ensembles of Virtual Agents on Technological Platforms. COJ Technical \& Scientific Research. 2 (4). 2020. Pages: 1-8.

[13] Evgeniy Bryndin. Transformation Modeling of Nanostructures by Cognitive Systems according to Big Smart Data. Chepter 3, Pages: 21-28. / Book "New Materials: Preparation, Properties and Applications in the Aspect of Nanotechnology". USA: Nova Science Publisher. 2020. 250 p

[14] Evgeniy Bryndin. Development of Artificial Intelligence by Ensembles of Virtual Agents with Mobile Interaction. Automation, Control and Intelligent Systems. Volume 8; Issue 1. 2020. Pages: 1-8.

[15] Evgeniy Bryndin. Formation of Technological Cognitive Reason with Artificial Intelligence in Virtual Space. Britain International of Exact Sciences Journal, Volume 2, Issue 2, May 2020. Page: 450-461.

[16] Bryndin E. G. Automation and artificial intelligence improve and accelerate the cleaning of territories. International Symposium "Current Directions of Scientific Research: Technologies, Quality and Safety." KGU. 2020. Pages: 103105.

[17] Bryndin E. G. Developing the behavioral and professional skills of sensitive cognitive robots as a security aspect. Collection X International Scientific Conference "ITSTANDARD 2020" - Moscow: Publishing House "Prospect," 2020. Page 303-310. 